\title{
Calculation of BER in multi-channel silicon optical interconnects: comparative analysis of strip and photonic crystal waveguides
}

\author{
Jie You ${ }^{\mathrm{a}}$, Spyros Lavdas ${ }^{\mathrm{a}}$, and Nicolae C. Panoiu ${ }^{\mathrm{a}}$ \\ ${ }^{a}$ Department of Electronic and Electrical Engineering, University College London, Torrington \\ Place, London WC1E 7JE, UK
}

\begin{abstract}
We present an effective approach to evaluate the performance of multi-channel silicon ( $\mathrm{Si}$ ) photonic systems. The system is composed of strip Si photonic waveguides ( $\mathrm{Si}-\mathrm{PhWs)} \mathrm{with} \mathrm{uniform} \mathrm{cross-section} \mathrm{or} \mathrm{photonic-crystal}$ $(\mathrm{PhC}) \mathrm{Si}$ waveguides $(\mathrm{Si}-\mathrm{PhCWs})$, combined with a set of direct-detection receivers. Moreover, the optical field in each channel is the superposition of a continuous-wave nonreturn-to-zero ON-OFF keying modulated signal and a white Gaussian noise. In order to characterize the optical signal propagation in the waveguides, an accurate mathematical model describing all relevant linear and nonlinear optical effects and its linearized version is employed. In addition, two semi-analytical methods - time- and frequency-domain Karhunen-Loève series expansion - are used to assess the system bit-error-rate (BER). Our analysis reveals that Si-PhCWs provide similar performance as Si-PhWs, but for $100 \times$ shorter length. Importantly, much worse BER is achieved in $\mathrm{Si}-\mathrm{PhCWs}$ when one operates in slow-light regime, due to the enhanced linear and nonlinear effects.
\end{abstract}

Keywords: BER evaluation, silicon interconnects, photonic crystal waveguides, Karhunen-Loève expansion, nonlinear pulse propagation

\section{INTRODUCTION}

Silicon optical interconnects have recently attracted great interest, due to primarily the significant potential to improve the performance and lower the cost of chip-scale optical networks. ${ }^{1,2}$ The main advantages of siliconon-insulator (SOI) platforms are perhaps the potential for system integration, which stems from the high-index contrast of $\mathrm{Si}$ waveguides, ${ }^{3}$ combined with the large optical nonlinearity of $\mathrm{Si}$, which allows for the design of chiplevel active photonic devices with new or improved functionalities. ${ }^{4}$ One promising approach to further increase the potential for system integration of the SOI platform is to employ photonic crystal (PhC) waveguides, ${ }^{5,6}$ which can provide access to slow-light (SL) propagation regime. ${ }^{7,8}$ In particular, a unique feature of the SL regime is that it enhances the linear and nonlinear optical effects, e.g., frequency dispersion, free-carriers (FCs) dispersion (FCD), and self- and cross-phase modulation (SPM, XPM), thus further decreasing the device footprint. In addition, due to great demand for large data transmission capacity and high data rates, wavelength-divisionmultiplexing (WDM) technology is key technology to be used in future communication systems. Therefore, it is particularly important to understand the characteristics of multi-channel optical signals propagating in photonic communication systems containing Si waveguides.

In this paper, a detailed investigation on the performance of multi-channel Si photonic systems is presented. Specifically, we assume that the WDM photonic system contains either a strip single-mode silicon photonic waveguide $(\mathrm{Si}-\mathrm{PhW})$ or a $\mathrm{Si} \mathrm{PhC}$ waveguide $(\mathrm{Si}-\mathrm{PhCW})$ as the $\mathrm{Si}$ optical interconnect, and direct-detection receivers to demultiplex and detect the optical signal in each channel, with $M$ channels considered. The photonic system considered in this work is schematically depicted in Fig. 1. Our analysis employs two theoretical models, namely a model that describes the multi-channel signal propagation in Si Waveguides, based on a system of coupled nonlinear Schrödinger equations (NLSEs), which fully incorporates linear and nonlinear optical effects, including FCD, FC absorption (FCA), SPM, XPM, two-photon absorption (TPA), FC dynamics, as well as the coupling between FCs, and the optical field and a simplified model, namely the linearized version of the full model. The bit-error rate (BER), the main quantity characterizing the performance of the communication link, is calculated by utilizing the time- and frequency-domain Karhunen-Loève series expansion (KLSE) method. ${ }^{9-11}$

Corresponding author: Nicolae C. Panoiu, E-mail: n.panoiu@ucl.ac.uk

Silicon Photonics and Photonic Integrated Circuits V, edited by Laurent Vivien, Lorenzo Pavesi, Stefano Pelli, Proc. of SPIE Vol. 9891, 989116 - @ 2016 SPIE · CCC code: 0277-786X/16/\$18 · doi: 10.1117/12.2223187 


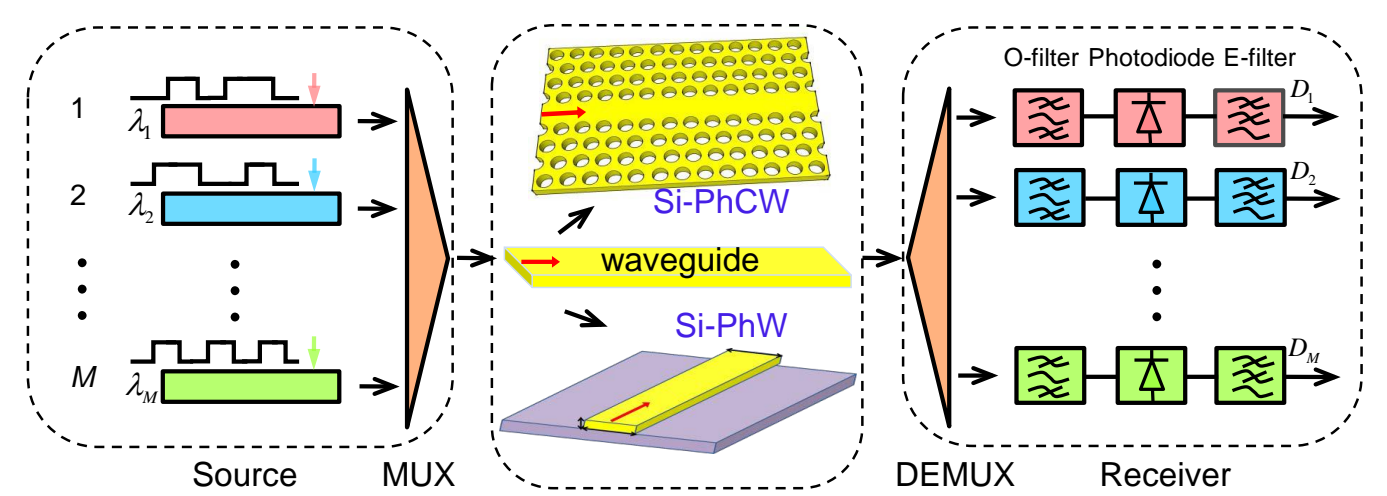

Figure 1: Schematic of the multi-channel photonic system, consisting of an array of lasers, MUX, silicon waveguide, DEMUX, and direct-detection receivers containing an optical band-pass filter, photodetector, and an electrical low-pass filter. The waveguide is either a strip waveguide with uniform cross-section or a $\mathrm{PhC}$ waveguide.

\section{THEORY OF MULTI-CHANNEL OPTICAL SIGNAL PROPAGATION}

Two theoretical models used to investigate the propagation of multi-wavelength optical signals in two types of $\mathrm{Si}$ waveguides are introduced in this section. The first approach is based on a system of coupled NLSEs whereas the second approach employs a linearized version of the full model, used in a parameter domain where the signal propagation is rigorously described. As illustrated in the central block of Fig. 1, the Si waveguide is either a single-mode $\mathrm{Si}-\mathrm{PhW}$ with uniform cross-section, buried in $\mathrm{SiO}_{2}$ cladding, or a $\mathrm{Si}-\mathrm{PhCW}$, consisting of a line defect in a two-dimensional honeycomb lattice of air holes in a Si slab. The optical modes of the latter waveguide possesses both fast-light (FL) and slow-light (SL) spectral regions. For convenience, we refer to the Si-PhCW when operated in these two regions as Si-PhCW-FL and Si-PhCW-SL, respectively.

\subsection{Full Theoretical Model}

The system of coupled equations describing multi-channel optical signal propagation and its interaction with FCs can be written as: ${ }^{12-14}$

$$
\begin{aligned}
j \frac{\partial u_{i}}{\partial z} & =-j\left(\frac{1}{v_{g, i}}-\frac{1}{v_{g, \text { ref }}}\right) \frac{\partial u_{i}}{\partial T}+\frac{\beta_{2, i}}{2} \frac{\partial^{2} u_{i}}{\partial T^{2}}-\frac{\omega_{i} \kappa_{i}}{n v_{g, i}} \delta n_{\mathrm{fc}} u_{i}-\frac{j c \kappa_{i}}{2 n v_{g, i}}\left(\alpha_{\mathrm{in}}+\alpha_{\mathrm{fc}}\right) u_{i}-\left(\gamma_{i}\left|u_{i}\right|^{2}+2 \sum_{k \neq i} \gamma_{i k}\left|u_{k}\right|^{2}\right) u_{i} \\
\frac{\partial N}{\partial t} & =-\frac{N}{\tau_{c}}+\sum_{i, k} C_{i k}\left|u_{i}\right|^{2}\left|u_{k}\right|^{2}
\end{aligned}
$$

Here, the $i$ th pulse envelope is $u_{i}(z, T), i=1, \ldots, M$, measured in $\sqrt{\mathrm{W}}, z$ is the propagation distance, $T$ represents the time in a reference system moving with velocity, $v_{g, \text { ref }}$, i.e. $T=t-z / v_{g \text {,ref }}$ where $t$ is the physical time, and $\omega_{i}$ is the $i$ th channel carrier frequency. The waveguide is characterized by the following parameters: $\kappa_{i}$ measures the overlap between the optical mode and the active area of the waveguide, $\alpha_{\mathrm{in}}$ is the intrinsic loss coefficient and is equal to $1 \mathrm{~dB} \mathrm{~cm}^{-1}\left(50 \mathrm{~dB} \mathrm{~cm}^{-1}\right)$ for $\mathrm{Si}-\mathrm{PhW}$ (Si-PhCW), $\tau_{c}$ is the FC relaxation time (in our analysis we assumed $\left.\tau_{c}=0.5 \mathrm{~ns}\right), \delta n_{\mathrm{fc}}$ and $\alpha_{\mathrm{fc}}$ are the FC-induced refractive index change and FC loss coefficient, respectively, and are given by $\delta n_{\mathrm{fc}}=\sigma_{n} N$ and $\alpha_{\mathrm{fc}}=\sigma_{\alpha} N$, where $N$ is the FC density, $\sigma_{\alpha}=1.45 \times 10^{-21}\left(\lambda / \lambda_{0}\right)^{2}$ (in units of $\mathrm{m}^{2}$ ), and $\sigma_{n}=\sigma\left(\lambda / \lambda_{0}\right)^{2}$ (in units of $\mathrm{m}^{3}$ ), with $\sigma$ and $\lambda_{0}=1550 \mathrm{~nm}$ being a power dependent coefficient ${ }^{15}$ and a reference wavelength, respectively. The nonlinear waveguide coefficients, $\gamma_{i}$ and $\gamma_{i j}$, describe SPM and XPM interactions, respectively, and the coefficient $C_{i k}$ in (1b) quantifies the rate at which the optical energy is transferred from the optical field to FCs; it is given by the following relations:

$$
C_{i k}=\left\{\begin{array}{c}
\frac{\gamma_{i}^{\prime \prime}}{\hbar \omega_{i} A_{\mathrm{nl}}}, \quad i=k, \\
\frac{4 \gamma_{i k}^{\prime \prime}}{\hbar\left(\omega_{i}+\omega_{k}\right) A_{\mathrm{nl}}}, \quad i \neq k .
\end{array}\right.
$$


Moreover, $A_{\mathrm{nl}}$ is the effective transverse area in which FCs are generated, with $A_{\mathrm{nl}}=w h\left(A_{\mathrm{nl}}=a h\right)$ in the $\mathrm{Si}-\mathrm{PhW}$ (Si-PhCW) case, and $\beta_{2, i}$ is the second-order dispersion coefficient.

\subsection{Linearized Model}

In order to linearize the system (1), we express the optical field in the $i$ th channel as the superposition between the corresponding optical signal and a Gaussian noise:

$$
u_{i}(z, T)=\left[\sqrt{P_{i}(z)}+a_{i}(z, T)\right] e^{-j \Phi_{i}(z)},
$$

where $P_{i}(z)$ stands for the $i$ th channel $\mathrm{CW}$ signal power, $a_{i}(z, T)$ is the complex additive Gaussian noise, and $\Phi_{i}(z)$ represents a global phase shift. The CW signals yield the steady-state FC density, $N_{s}$, with the expression derived from (1b): $N_{s}(z)=\sum_{i, k} \xi_{i k} P_{i}(z) P_{k}(z)$, where $\xi_{i k}=\tau_{c} C_{i k}$.

To linearize (1), we first substitute (3) into (1) and then discard all quadratic and higher-order terms in $a_{i}(z, T)$. This leads to the following system of equations:

$$
\begin{aligned}
\frac{d P_{i}}{d z}= & -\frac{c \kappa_{i}}{n v_{g, i}}\left(\alpha_{\mathrm{in}}+\sigma_{\alpha} N_{s}\right) P_{i}-2\left(\gamma_{i}^{\prime \prime} P_{i}+2 \sum_{k \neq i} \gamma_{i k}^{\prime \prime} P_{k}\right) P_{i} \\
\frac{d \Phi_{i}}{d z}= & -\frac{\omega_{i} \kappa_{i}}{n v_{g, i}} \sigma_{n} N_{s}-\gamma_{i}^{\prime} P_{i}-2 \sum_{k \neq i} \gamma_{i k}^{\prime} P_{k}, \\
\frac{d A_{i}^{\prime}}{d z}= & j\left(\frac{1}{v_{g, i}}-\frac{1}{v_{g, \text { ref }}}\right) \Omega_{i} A_{i}^{\prime}-\frac{\beta_{2, i}}{2} \Omega_{i}^{2} A_{i}^{\prime \prime}-\frac{c \kappa_{i}}{2 n v_{g, i}}\left[\left(\alpha_{\text {in }}+\sigma_{\alpha} N_{s}\right) A_{i}^{\prime}+2 \sigma_{\alpha} \sqrt{P_{i}}\right. \\
& \left.\sum_{k l} P_{k} \sqrt{P_{l}}\left(\xi_{k l}+\xi_{l k}\right) A_{l}^{\prime}\right]-3 \gamma_{i}^{\prime \prime} P_{i} A_{i}^{\prime}-2 \sum_{k \neq i} \sqrt{P_{k}}\left(\gamma_{i k}^{\prime \prime} \sqrt{P_{k}} A_{i}^{\prime}+2 \gamma_{i k}^{\prime \prime} \sqrt{P_{i}} A_{k}^{\prime}\right), \\
\frac{d A_{i}^{\prime \prime}}{d z}= & j\left(\frac{1}{v_{g, i}}-\frac{1}{v_{g, \text { ref }}}\right) \Omega_{i} A_{i}^{\prime \prime}+\frac{\beta_{2, j}}{2} \Omega_{i}^{2} A_{i}^{\prime}+2 \frac{\omega_{i} \kappa_{i}}{n v_{g, i}} \sigma_{n} \sqrt{P_{i}} \sum_{k l} P_{k} \sqrt{P_{l}}\left(\xi_{k l}+\xi_{l k}\right) A_{l}^{\prime} \\
& -\frac{c \kappa_{i}}{2 n v_{g, i}}\left(\alpha_{\text {in }}+\sigma_{\alpha} N_{s}\right) A_{i}^{\prime \prime}+\left(2 \gamma_{i}^{\prime} A_{i}^{\prime}-\gamma_{i}^{\prime \prime} A_{i}^{\prime \prime}\right) P_{i}-2 \sum_{k \neq i} \sqrt{P_{k}}\left(\gamma_{i k}^{\prime \prime} \sqrt{P_{k}} A_{i}^{\prime \prime}-2 \gamma_{i k}^{\prime} \sqrt{P_{i}} A_{k}^{\prime}\right) .
\end{aligned}
$$

Here, $\Omega_{i}=\omega-\omega_{i}, A_{i}^{\prime}\left(z, \Omega_{i}\right)=\mathcal{F}\left\{a_{i}^{\prime}(z, T)\right\}$, and $A_{i}^{\prime \prime}\left(z, \Omega_{i}\right)=\mathcal{F}\left\{a_{i}^{\prime \prime}(z, T)\right\}$, with $a_{i}^{\prime}(z, T)$ and $a_{i}^{\prime \prime}(z, T)$ being in-phase and quadrature noise components, respectively. Importantly, the FC-mediated interactions among different channels result in an interchannel cross-talk mechanism, revealed by the terms proportional to $N_{s}$ in (4a) and (4b).

\section{KARHUNEN-LOĖVE SERIES EXPANSION METHOD FOR BER CALCULATION}

To implement the KLSE method, two assumptions are made: firstly, the direct-detection receivers for each channel are identical and secondly, the demultiplexer will not induce additional noise correlations.

\subsection{Time Domain Formulation}

For the time domain KLSE method, we define $r_{1}(t)=S+a_{i}(t)+j a_{q}(t)$ as the receiver input optical signal, where $a_{i}(t)$ and $a_{q}(t)$ are the in-phase and quadrature noise components, respectively, and $S$ is the amplitude of the CW signal. The "0" ("1") bit is represented by $S=0\left(S^{2}=P\right)$. After the optical filter described by the response function, $h_{o}(t)$, the signal can be written as $r_{2}(t)=S+v_{i}(t)+j v_{q}(t)$, where $v(t)=h_{o}(t) \otimes a(t)$. Moreover, after passing through the ideal square-law photodetector and the electrical filter described by the response function, $h_{e}(t)$, the electrical signal is obtained as:

$$
y(t)=\int_{-\infty}^{\infty} h_{e}(t)\left\{\left[S+v_{i}\left(t-t^{\prime}\right)\right]^{2}+v_{q}^{2}\left(t-t^{\prime}\right)\right\} d t^{\prime} .
$$


In order to determine the BER, firstly, we need to expand the signal $S$ and noise components $v_{i}, v_{q}$ in KL series, with deterministic functions $\left\{\phi_{\alpha}^{k}(t)\right\}$ and $\left\{\psi_{\alpha}^{k}(t)\right\}$, orthonormal to the weight function $h_{e}(t):{ }^{16}$

$$
\begin{aligned}
& S=\sum_{\alpha \geq 1} s_{\alpha} \phi_{\alpha}(t), \\
& v_{i}(t)=\sum_{\alpha \geq 1} p_{\alpha} \phi_{\alpha}(t), \\
& v_{q}(t)=\sum_{\alpha \geq 1} q_{\alpha} \psi_{\alpha}(t),
\end{aligned}
$$

where for convenience the channel index, $k$, has been dropped. Then we calculate the correlation matrix using a well-known algorithm ${ }^{17}$ and from it the moment-generating function (MGF), $\Psi_{y}(\zeta)$, of the random variable $y$ defined by (5) is calculated as: ${ }^{18}$

$$
\Psi_{y}(\zeta)=\mathbb{E}\left\{e^{-\zeta y}\right\}=\prod_{\alpha=1}^{2 M} \frac{\exp \left(-\frac{\left|\eta_{\alpha}\right|^{2} \zeta}{1+2 \delta_{\alpha} \zeta}\right)}{\sqrt{1+2 \delta_{\alpha} \zeta}} .
$$

Here, $\delta_{\alpha}$ are the eigenvalues of the correlation matrix and $\eta_{\alpha}$ are the expectation values of the signal at the output of the optical filter. Finally, we evaluate the system BER defined by the following relation:

$$
\mathcal{P}=\frac{1}{2}\left[\mathcal{P}\left(y>y_{t h} \mid S=0\right)+\mathcal{P}\left(y<y_{t h} \mid S=\sqrt{P}\right)\right],
$$

where $y_{t h}$ is the decision threshold. Implementing the Riemann-Fourier inversion formula of the MGF, we can measure these probabilities as follows: ${ }^{19}$

$$
\begin{aligned}
& \mathcal{P}\left(y>y_{t h} \mid S=0\right)=-\int_{-|\bar{\zeta}|-\infty}^{-|\bar{\zeta}|+\infty} \frac{\Psi_{y}(\zeta \mid S=0)}{2 \pi j \zeta} e^{\zeta y_{t h}} d \zeta, \\
& \mathcal{P}\left(y<y_{t h} \mid S=\sqrt{P}\right)=\int_{|\bar{\zeta}|-\infty}^{|\bar{\zeta}|+\infty} \frac{\Psi_{y}(\zeta \mid S=\sqrt{P})}{2 \pi j \zeta} e^{\zeta y_{t h}} d \zeta,
\end{aligned}
$$

where $\bar{\zeta}$ is a real constant that defines the integration path in the complex plane, $\zeta$.

\subsection{Frequency Domain Formulation}

In this subsection we briefly outline the frequency domain method. ${ }^{10,11,20}$ Thus, in this case the phtocurrent (5) can be expressed as,

$$
y(t)=\int_{-\infty}^{\infty} \int_{-\infty}^{\infty} Y^{*}\left(f_{1}\right) K\left(f_{1}, f_{2}\right) Y\left(f_{2}\right) e^{2 \pi j\left(f_{2}-f_{1}\right) t} d f_{1} d f_{2}
$$
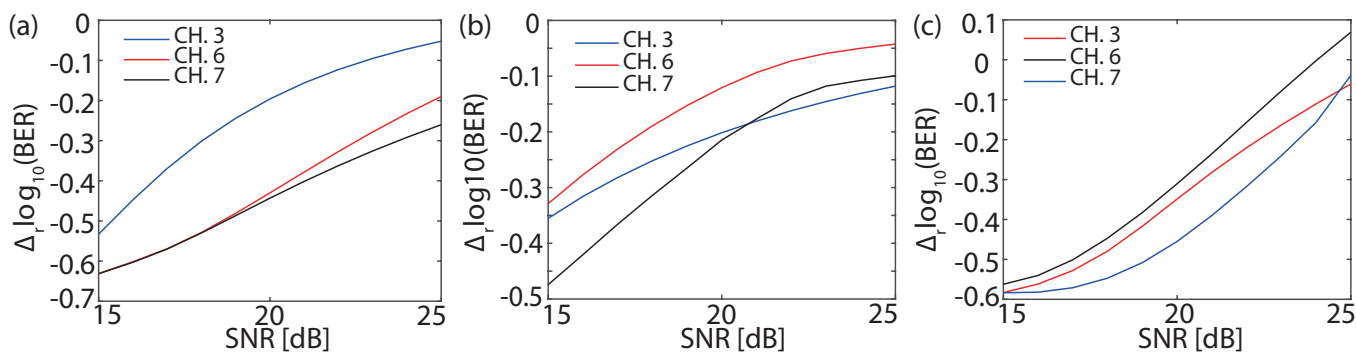

Figure 2: Comparison of the system BER calculated via the time- and frequency-domain KLSE method. The 8-channel Si-PhW (a), Si-PhCW operating in the FL regime (b), and a Si-PhCW operating in the SL regime (c) systems are considered. The input signal power in each channel is $P=5 \mathrm{~mW}$. The relative difference of BER calculated via the two methods is quantified by $\Delta_{r} \log _{10}(\mathrm{BER})=\left[\log _{10}(\mathrm{BER})_{F D}-\log _{10}(\mathrm{BER})_{T D}\right] / \log _{10}(\mathrm{BER})_{F D}$. 
where the Hermitian kernel in this equation is given by:

$$
K\left(f_{1}, f_{2}\right)=H_{o}^{*}\left(f_{1}\right) H_{o}\left(f_{2}\right) H_{e}\left(f_{2}-f_{1}\right) .
$$

Here, $H_{o}(f)=\mathcal{F}\left\{h_{o}(t)\right\}$ and $H_{e}(f)=\mathcal{F}\left\{h_{e}(t)\right\}$, are frequency-domain response functions of the optical and electrical filters, respectively. Similarly, $Y(f)$ is expanded in a full basis of eigenfunctions of the Fredholm integral equation, which is defined by the kernel $K\left(f_{1}, f_{2}\right)$. After obtaining the correlation matrix, one calculates the MGF of the statistical variable $y$ and the BER using a procedure similar to the one employed in the time-domain.

\subsection{Comparison Between the Two Methods}

To verify the validity of the two methods we compared their predictions, the results being summarized in Fig. 2. In this figure, we plot the dependence on the SNR of the relative difference of the BER calculated using the two KLSE methods, where this relative BER difference is defined as $\Delta_{r} \log _{10}(\mathrm{BER})=\left[\log _{10}(\mathrm{BER})_{F D}-\right.$ $\left.\log _{10}(\mathrm{BER})_{T D}\right] / \log _{10}(\mathrm{BER})_{F D}$. We performed this analysis for three channels, namely for channel 3, 6, and 7 , in an 8-channel Si-PhW, Si-PhCW-FL, and Si-PhCW-SL system. The plots in Fig. 2 show that the predictions of the two methods agree well, particularly at large values of the SNR.

\section{ANALYSIS OF SYSTEM PERFORMANCE}

In this section we consider generic multi-channel photonic systems containing both types of Si waveguides, namely $\mathrm{Si}-\mathrm{PhW}$ and $\mathrm{Si}-\mathrm{PhCW}$. In both cases the bit window is $T_{0}=100 \mathrm{ps}$, the channel spacing is $\Delta \omega=25 \mathrm{GHz}$, and the reference wavelength for the Si-PhW, Si-PhCW-FL, and Si-PhCW-SL systems is $1550 \mathrm{~nm}, 1550 \mathrm{~nm}$, and $1523.9 \mathrm{~nm}$, respectively. Also, the dispersion and nonlinear waveguide coefficients at the reference wavelength are: $\beta_{2}=0.5 \mathrm{ps}^{2} \mathrm{~m}^{-1}$ and $\gamma=(166.8+50.8 j) \mathrm{W}^{-1} \mathrm{~m}^{-1}$ for the Si-PhW, $\beta_{2}=-3.3 \times 10^{2} \mathrm{ps}^{2} \mathrm{~m}^{-1}$ and $\gamma=$ $(750.1+228.6 j) \mathrm{W}^{-1} \mathrm{~m}^{-1}$ for Si-PhCW-FL, and $\beta_{2}=-4.3 \times 10^{4} \mathrm{ps}^{2} \mathrm{~m}^{-1}$ and $\gamma=(6.94+2.12 j) \times 10^{3} \mathrm{~W}^{-1} \mathrm{~m}^{-1}$ for the Si-PhCW-SL. The length of the Si-PhW (Si-PhCW) is $L=5 \mathrm{~cm}(L=500 \mu \mathrm{m})$, unless otherwise stated.

The first system parameter considered in our study is the optical power of the signal, since it is perhaps the physical quantity that most significantly influences the photonic system performance. The reason for this is that larger optical power leads to stronger nonlinear optical effects in the waveguide and a larger amount of generated FCs, both effects modifying the optical properties of the waveguide. Thus, the dependence of the transmission BER of the three 8-channel systems just described on the input power in each channel is presented in Fig. 3. In this figure, we show the BER corresponding to channel 1 and channel 6. We choose different channels because in the 8-channel Si-PhCW-SL system, the dispersion characteristics of the optical guiding mode imply that the
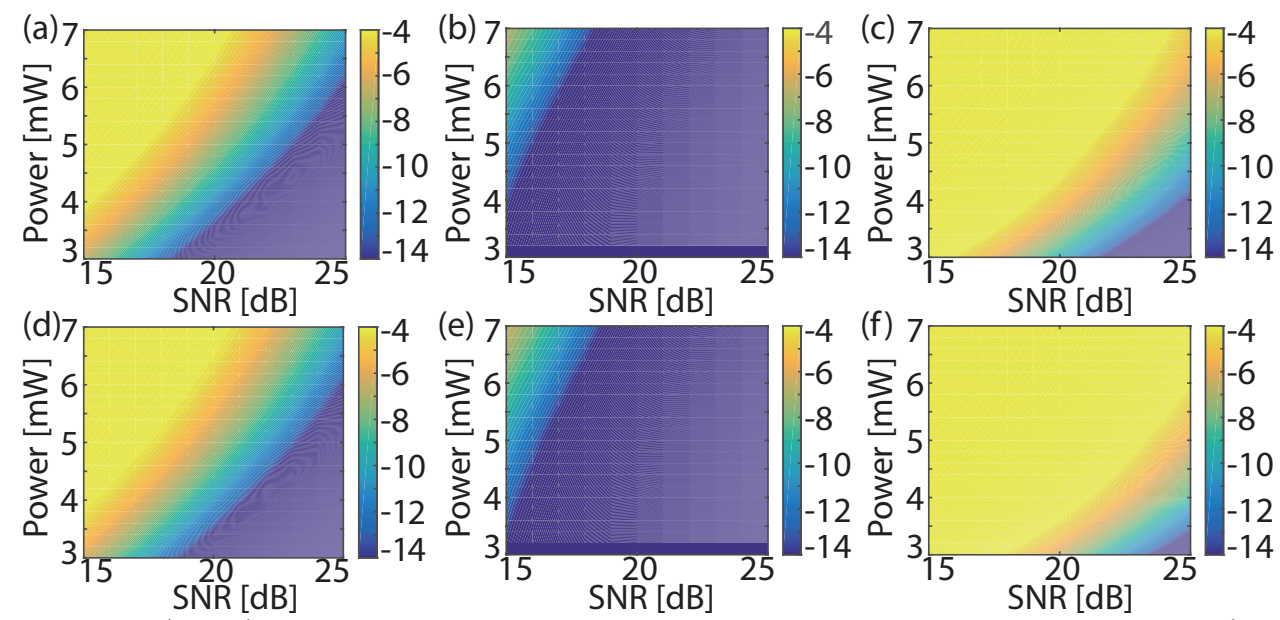

Figure 3: Maps of $\log _{10}(\mathrm{BER})$ vs. power and SNR, calculated for a 8-channel Si-PhW system (left), a Si-PhCW operating in the FL regime system (middle), and a Si-PhCW operating in the SL regime system (right). The top and bottom panels correspond to channel 1 and channel 6 , respectively. 
larger is the channel index, the smaller is the group-velocity and consequently the more enhanced are the linear and nonlinear optical effect. As a result, in such a dispersive system the quality of the transmitted signal strongly depends on the channel index. However, this analysis is not valid in the cases of the Si-PhW and Si-PhCW-FL systems, a conclusion validated by the small difference between the data plotted in Figs. 3(a) and 3(d), and in Figs. 3(b) and 3(e).

Another key finding of this investigation is that the system BER increases with the input power, provided that the value of the SNR is kept constant. In addition, the Si-PhW system provides relatively small transmission BER either when the input power is small or when the value of SNR is large. Moreover, the Si-PhCW-FL system has the best performance, since it provides a larger parameter space where the system BER is smaller than an upper-bound limit commonly used in optical communications systems, namely BER $<10^{-9}$. This is true despite the fact that the length of this waveguide is $100 \times$ shorter than that of the Si-PhW. On the other hand, when the same $\mathrm{PhC}$ waveguide is operated in the SL regime it provides the worst signal quality as compared to the other two system configurations. One way to improve the performance of this system is to decrease the input power in each channel, to a level that ensures that the BER is smaller than $10^{-9}$.

The waveguide length is another key parameter that strongly influences the transmission BER, so that it is worth to investigate this dependence in order to provide guidance to the design of highly efficient Si waveguides for on-chip optical interconnects. To this end, different lengths of both Si-PhW and Si-PhCW waveguides were considered, but the ratio of these two waveguide lengths was fixed to 100, with the aim of providing a clear comparison among different systems by only using one variable - the waveguide length. The dependence of the system BER versus the waveguide length, in the case of the three 8-channel systems described above and for the signal power, $P=5 \mathrm{~mW}$, is presented in Fig. 4. A key feature illustrated by this figure is that for all three systems the BER decreases when the waveguide length decreases. In addition, the Si-PhCW operated in the FL regime can provide the best performance among the three cases investigated, as in this case the BER is smaller than the threshold of $10^{-9}$ in almost the entire parameter space covered by Figs. 4(b) and 4(e).
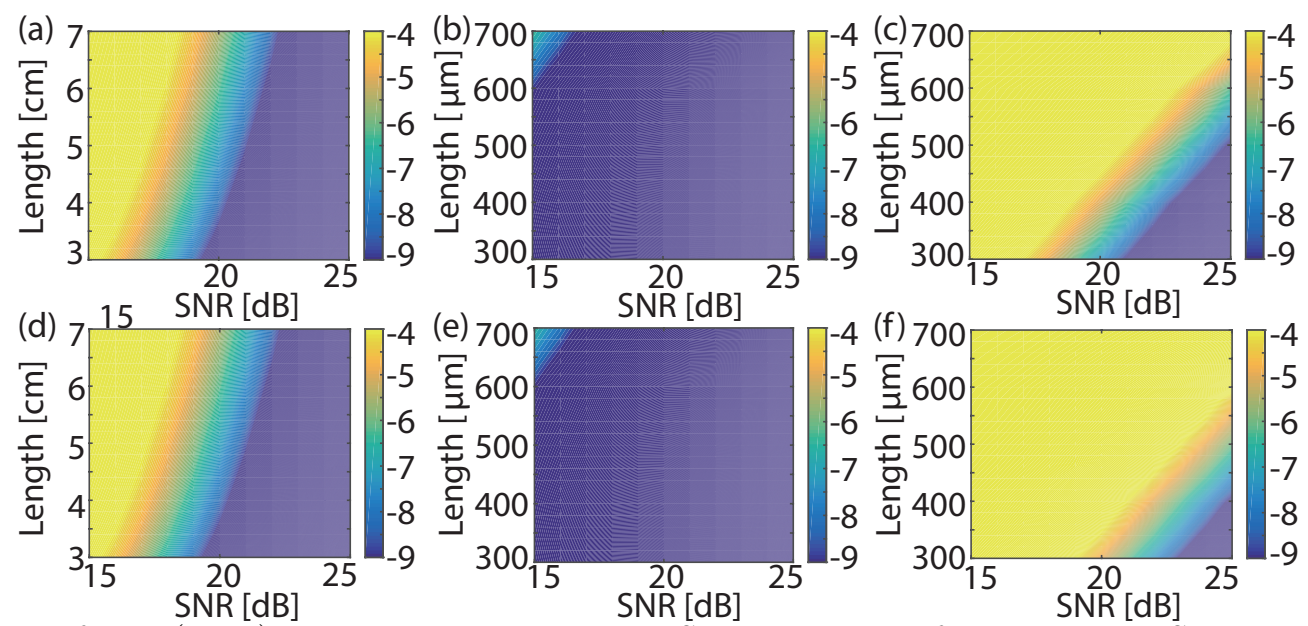

Figure 4: Maps of $\log _{10}$ (BER) vs. waveguide length and SNR, calculated for a 8-channel Si-PhW system (left), a $\mathrm{Si}-\mathrm{PhCW}$ operating in the FL regime system (middle), and a Si-PhCW operating in the SL regime system (right). The top and bottom panels correspond to channel 1 and channel 6, respectively. The input power in each channel is $P=5 \mathrm{~mW}$.

\section{CONCLUSIONS}

In conclusion, a detailed analysis of bit-error rate in multi-channel photonic systems containing silicon waveguides was presented. Both a strip waveguide with uniform cross-section and a photonic crystal waveguide were considered as two generic types of such optical interconnects. Moreover, we extended our analysis to an important physical regime pertaining to photonic crystal waveguides, namely slow-light propagation, where both linear and nonlinear optical effects are strongly enhanced. Our analysis of the bit-error rate in these photonic 
systems revealed that reduced device footprint can be achieved by using photonic crystal waveguides when they operate in the fast-light regime. On the other hand, significant degradation of the system performance was observed when the photonic crystal waveguide was operated in the slow-light regime at similar power levels, because the enhanced linear and nonlinear optical effects induce large inter-channel cross-talk. Importantly, the theoretical models introduced in this study can also be applied to other, more complex devices of practical interest. For example, other photonic devices can be added in the system by properly modifying the theoretical models describing the optical field dynamics in the silicon waveguides. This shows that, among other things, our work is particularly relevant to the development of future chip-scale optical interconnects implemented on the silicon-on-insulator platform.

\section{REFERENCES}

[1] Park, H., Fang, A. W., Kodama, S., and Bowers, J. E., "Hybrid silicon evanescent laser fabricated with a silicon waveguide and III-V offset quantum wells," Opt. Express 13(23), 9460-9464 (2005).

[2] Goodman, J. W., Leonberger, F. I., Kung, S.-Y., and Athale, R. A., "Optical interconnections for VLSI systems," Proc. IEEE 72(7), 850-866 (1984).

[3] Lee, K. K., Lim, D. R., Luan, H.-C., Agarwal, A., Foresi, J., and Kimerling, L. C., "Effect of size and roughness on light transmission in a si/sio2 waveguide: Experiments and model," Appl. Phys. Lett. 77(11), 1617-1619 (2000).

[4] Osgood, R. M., Panoiu, N. C., Dadap, J. I., Liu, X., Chen, X., Hsieh, I.-W., Dulkeith, E., Green, W. M., and Vlasov, Y. A., "Engineering nonlinearities in nanoscale optical systems: physics and applications in dispersion-engineered silicon nanophotonic wires," Adv. Opt. Photon. 1(1), 162-235 (2009).

[5] Mekis, A., Chen, J., Kurland, I., Fan, S., Villeneuve, P. R., and Joannopoulos, J., "High transmission through sharp bends in photonic crystal waveguides," Phys. Rev. Lett. 77(18), 3787 (1996).

[6] Lin, S.-Y., Chow, E., Hietala, V., Villeneuve, P. R., and Joannopoulos, J., "Experimental demonstration of guiding and bending of electromagnetic waves in a photonic crystal," Science 282(5387), 274-276 (1998).

[7] Baba, T., "Slow light in photonic crystals," Nat. Photon. 2(8), 465-473 (2008).

[8] Krauss, T. F., "Why do we need slow light?," Nat. Photon. 2(8), 448-450 (2008).

[9] Papoulis, A. and Pillai, S. U., [Probability, random variables, and stochastic processes], McGraw-Hill (2002).

[10] Mafi, A. and Raghavan, S., "Nonlinear phase noise in optical communication systems using eigenfunction expansion method," Opt. Eng. 50(5), 055003 (2011).

[11] Forestieri, E. and Secondini, M., "On the error probability evaluation in lightwave systems with optical amplification," J. Lightw. Technol. 27(6), 706-717 (2009).

[12] Chen, X., Panoiu, N. C., and Osgood Jr, R. M., "Theory of raman-mediated pulsed amplification in siliconwire waveguides," IEEE J. Quantum Electron. 42(2), 160-170 (2006).

[13] Panoiu, N. C., McMillan, J. F., and Wong, C. W., "Theoretical analysis of pulse dynamics in silicon photonic crystal wire waveguides," IEEE J. Sel. Topics Quantum Electron. 16(1), 257-266 (2010).

[14] Lavdas, S. and Panoiu, N. C., "Theory of pulsed four-wave-mixing in one-dimensional silicon photonic crystal slab waveguides," Phys. Rev. B (to be published).

[15] Lin, Q., Painter, O. J., and Agrawal, G. P., "Nonlinear optical phenomena in silicon waveguides: modeling and applications," Opt. Express 15(25), 16604-16644 (2007).

[16] Helstrom, C. W., [Statistical Theory of Signal Detection], Pergamon, New York (1968).

[17] Bosco, G., Carena, A., Curri, V., Gaudino, R., Poggiolini, P., and Benedetto, S., "A novel analytical approach to the evaluation of the impact of fiber parametric gain on the bit error rate," IEEE Trans. Commun. 49(12), 2154-2163 (2001).

[18] Mathai, A. M. and Provost, S. B., [Quadratic forms in random variables: theory and applications], M. Dekker, New York (1992).

[19] Helstrom, C., "Distribution of the filtered output of a quadratic rectifier computed by numerical contour integration," IEEE Trans. Inf. Theory 32(4), 450-463 (1986).

[20] You, J., Lavdas, S., and Panoiu, N. C., "Theoretical comparative analysis of ber in multi-channel systems with strip and photonic crystal silicon waveguides," IEEE J. Sel. Topics Quantum Electron. 22(2), 4400810 (2016). 